\title{
Laser Microdissection as a New Approach to Prefertilization Genetic Diagnosis
}

\author{
Annette Clement-Sengewald ${ }^{a}$ Tina Buchholz $^{b}$ Karin Schütze ${ }^{c}$ \\ al. Frauenklinik der Universität, and ${ }^{\mathrm{b}}$ Klinik und Poliklinik für Frauenheilkunde und Geburtshilfe, Klinikum \\ Grosshadern, Ludwig Maximilians University, Munich, and 'PALM Mikrolaser Technologie, Bernried, Germany
}

\section{Key Words}

Laser microdissection - Laser pressure catapulting • Laser tweezers - Laser trap - Polar body analysis . Prefertilization, genetic diagnosis - UV-A laser beam

\begin{abstract}
The genetic status of oocytes can be determined by polar body (PB) analysis. Following PB extraction, a genetic evaluation is performed. As each PB contains the complementary genetic material of the oocyte, PB analysis reveals information about its genetic status. Genetically altered oocytes may then be excluded from in vitro fertilization. The aim of our study was to evaluate laser microdissection as a tool for PB extraction purposes. Compared to the PB extraction with a sharp-ending pipette only, we could show that laser microdissection of the zona pellucida (laser zona drilling) with a UV-A laser and subsequent extraction with a blunt-ending pipette decreases the degeneration rate of oocytes. It is shown that laser pressure catapulting of extracted PB enables their contact-free transfer into tubes, thus decreasing the risk of contamination for further analysis.
\end{abstract}

Copyright $(2001$ S. Karger AG, Base

\section{Introduction}

Polar body (PB) analysis is a helpful procedure for the identification of oocytes bearing chromosomal disorders or mutations. As these altered oocytes will result in a failure of fertilization, abnormal embryo development, failure of implantation and early or late fetal loss, it would be necessary to identify these oocytes and exclude them from in vitro fertilization (IVF; prefertilization genetic diagnosis). As older women have a higher risk of chromosomal aberrations, a prefertilization genetic diagnosis may help to increase pregnancy- and baby-take-home rates especially in these patients. Furthermore, PB analysis may help patients with a known chromosomal disorder or mutation with otherwise minimal chances of a healthy baby. For PB analysis, $\mathrm{PB}$ have to be extracted from the perivitelline space of the oocyte. This has to be done by micromanipulation, either mechanically with micropipettes [1,2] or with a combination of different methods, i.e. chemical treatment of the zona pellucida and subsequent mechanical withdrawal of the PB [3]. All methods bear the risk of damage to the PB or the oocyte. Therefore, optimization of the extraction method is one of the main tasks in prefertilization genetic diagnosis. As a degenerated PB may result in a false interpretation in subsequent genetic analysis [fluorescence in situ hybridization (FISH), single-cell polymerase chain reaction (PCR)], careful handling is a

\begin{tabular}{|c|c|}
\hline KARGER & $\begin{array}{l}\text { (C) } 2001 \text { S. Karger AG, Basel } \\
1015-2008 / 00 / 0685-0232 \$ 17.50 / 0\end{array}$ \\
\hline $\begin{array}{l}\text { Fax + } 41613061234 \\
\text { E-Mail karger@karger.ch } \\
\text { www.karger.com }\end{array}$ & $\begin{array}{l}\text { Accessible online at: } \\
\text { www.karger.com/journals/pat }\end{array}$ \\
\hline
\end{tabular}

Dr. Annette Clement-Sengewald

I. Frauenklinik der Universität

Maistrasse 11

D-80337 München (Germany)

Tel. +49 895160 4308, Fax +49 895160 4149, E-Mail a-s@sengewald.m.uunet.de 
prerequisite for successful analysis. On the other hand, damage of the oocyte will alter the fertilization process and subsequent embryo development.

For a long time laser light has been used to manipulate cells or even cell fragments [4]. As laser beams may now be guided through optical lenses, it is possible to exactly focus the laser beam to less than $1 \mu \mathrm{m}$ in diameter. In assisted reproductive technologies, laser light was used for manipulation of sperm and oocytes, thus supporting or replacing conventional manipulation methods [5-7].

First experiments in oocytes to microdissect the zona pellucida (laser zona drilling) were performed in order to facilitate sperm penetration $[6,8]$. In the last years laser zona drilling has been reported to facilitate embryo hatching $[9,10]$. In 1998, a diode laser was used to drill a hole into the zona pellucida of mouse oocytes and to subsequently extract PB by means of a blunt-ended pipette [11].

In this study we compared the conventional PB extraction method, using a sharp-ended pipette, with the combined extraction method of microdissection with a UV-A laser beam and PB extraction with a blunt-ended pipette. Furthermore, we tried to use laser tweezers for trapping and movement of $\mathrm{PB}$ to find out whether laser tweezers might be helpful for PB extraction.

In 1999, a new method for a noncontact, lasermediated capture of single cells was published, the socalled laser pressure catapulting [12]. After isolation by laser microdissection, membrane-bound single cells were catapulted into PCR tubes. It was shown that this method did not impair subsequent DNA and RNA analysis.

For single-cell PCR contamination is very critical. Therefore, we tested the laser pressure catapulting method for the noncontact transfer of extracted PBs into PCR tubes.

\section{Materials and Methods}

\section{Human Oocytes}

For the experiments human oocytes were used which had failed to fertilize during the routine IVF program. Furthermore, surplus oocytes and oocytes showing abnormal signs of fertilization (one or three pronuclei) were used. Thus, oocytes were available 24 and $48 \mathrm{~h}$ after their retrieval. The experiments were accepted by the Ethics Committee of the Ludwig Maximilians University (No. 182/98). Patients who agreed with the use of their oocytes for the experiments gave us their written content.

\section{Laser Equipment}

For all experiments a PALM ${ }^{\circledR}$ Robot-CombiSystem was used (PALM Microlaser Technologies, Bernried, Germany), consisting of a pulsed, UV-A nitrogen laser $(337 \mathrm{~nm})$ for laser microdissection

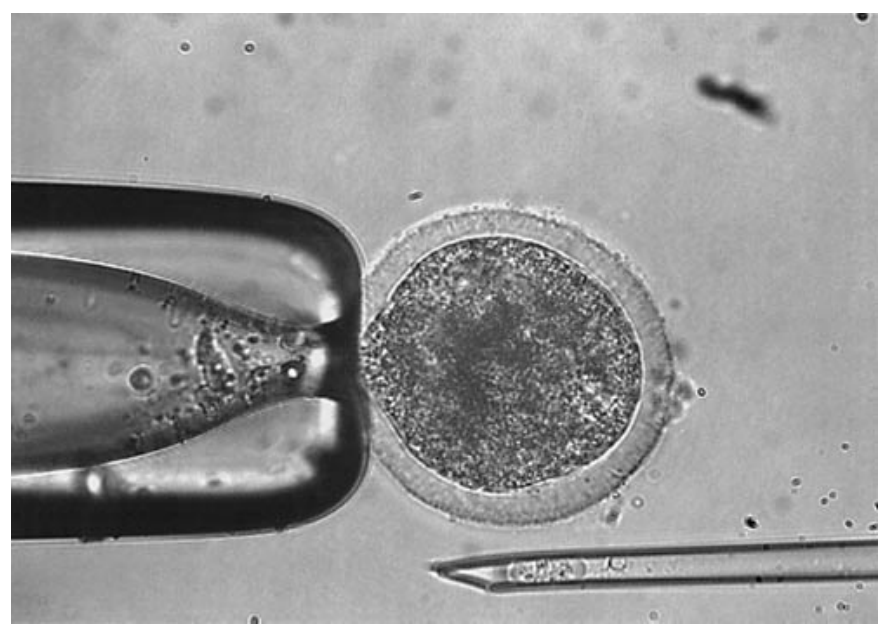

Fig. 1. $P B$ extraction with a sharp-ended pipette (bovine oocyte). The oocyte was fixed with the PB in the 6 o'clock position. The pipette was inserted in the 5 o'clock position, and the PB was carefully sucked into the pipette. After withdrawal, the PB can be seen in the extraction pipette.

and a continuous-wave, diode-pumped Nd:YAG laser $(1064 \mathrm{~nm}$, $2000 \mathrm{~mW}$ ) for laser tweezers purposes. The two laser beams were coupled into a microscope and their focus was adjusted to the optical focus of the microscope. All experiments were performed on a thin $(0.17 \mathrm{~mm})$ glass slide in a drop of $50 \mu \mathrm{l}$ of manipulation medium (Ham's F10 medium $+10 \%$ human albumin; ICN Biochemicals, Aurora, Ohio, USA). A 100-fold objective was used for all experiments.

\section{Micromanipulation}

Holding pipettes were pulled by hand, broken and subsequently fire-polished to an outer diameter of $150-180 \mu \mathrm{m}$ and an inner diameter of 20-30 $\mu \mathrm{m}$. For removal of the PB with a sharp-ended pipette, a borosilicate glass tube was pulled and broken in order to obtain an outer diameter of $15-20 \mu \mathrm{m}$ at the tip. The tip was ground at an angle of $45^{\circ}$, and was subsequently washed in acid and water. Then, an additional peak was pulled to the tip with the help of a microforge. This peak guaranteed easy penetration of the zona pellucida. The blunt-ended pipette was pulled to an outer diameter of $15-20 \mu \mathrm{m}$, broken and fire-polished. For micromanipulation, the pipettes were connected to an air-filled (holding pipette) or paraffin oil-filled (extraction pipette) tube system. Their motion was controlled by mechanical micromanipulators.

For PB removal with the sharp-ended pipette, the oocyte was fixed by the holding pipette. The position of the PB was carefully considered to enable pipette penetration without injuring the oocyte. Therefore, the PB was in the 1 or 5 o'clock position (fig. 1), and the extraction pipette was inserted in the 2 or 4 o'clock position. The pipette was pushed between the PB and the oocyte, and the PB was carefully sucked into the pipette. After withdrawal of the pipette, the PB was released into a separate drop of medium. The oocyte was inspected 5 and 30 min after micromanipulation in order to evaluate its morphology. 

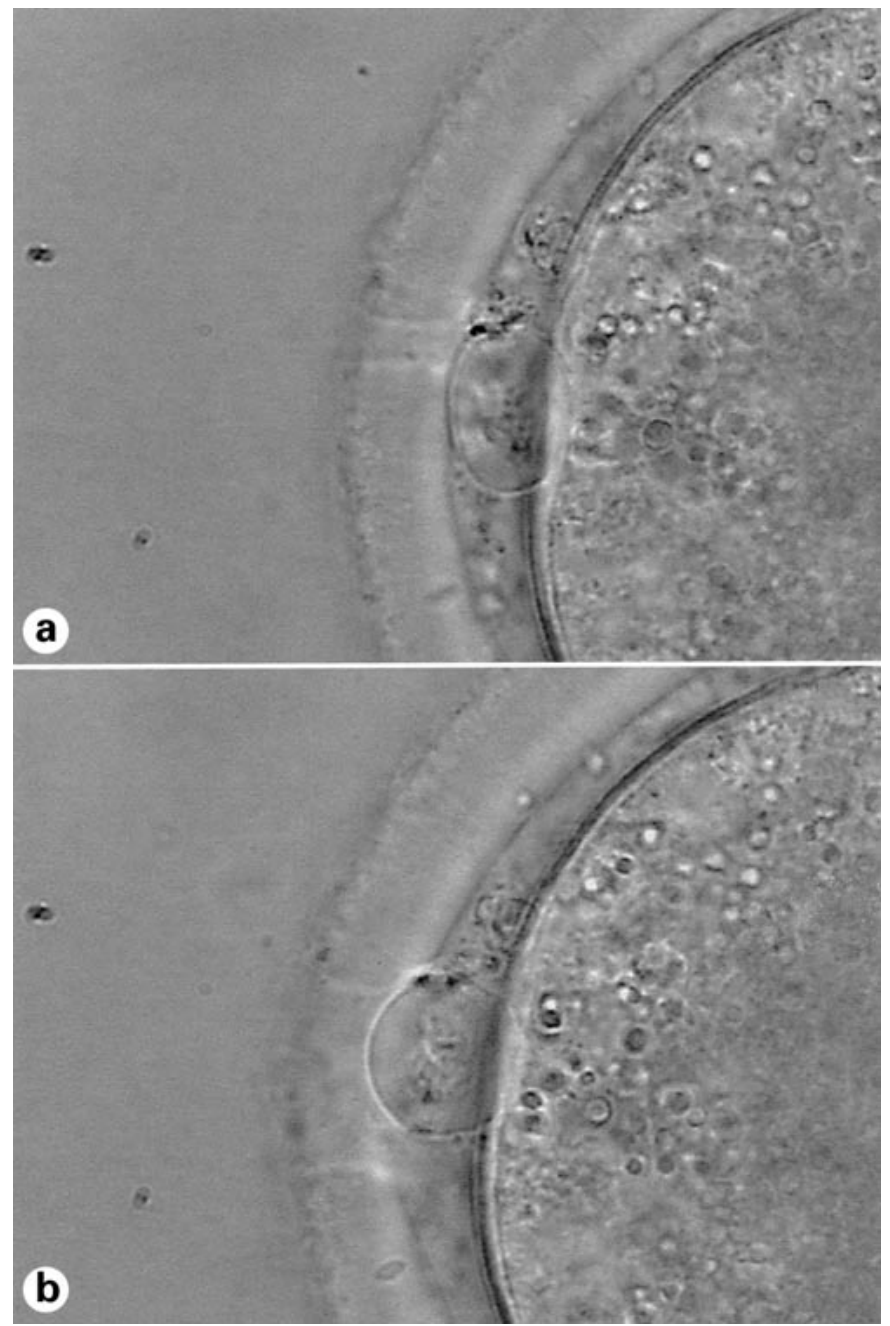

Fig. 2. Laser microdissection of the zona pellucida (laser zona drilling). a A small channel is drilled by moving the oocyte backwards and forewards into the UV-A microbeam. b After laser zona drilling, the hole was drilled slightly bigger than the size of the PB.

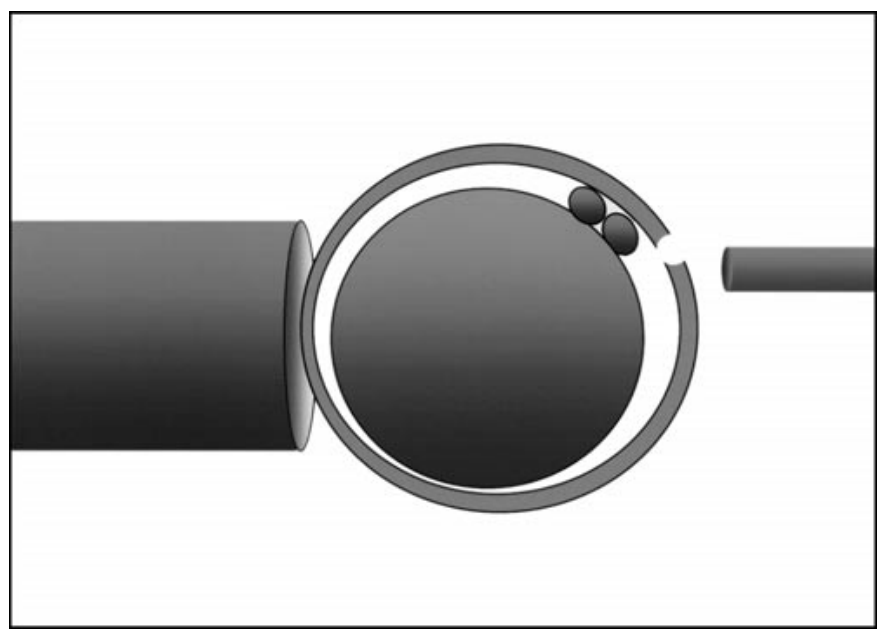

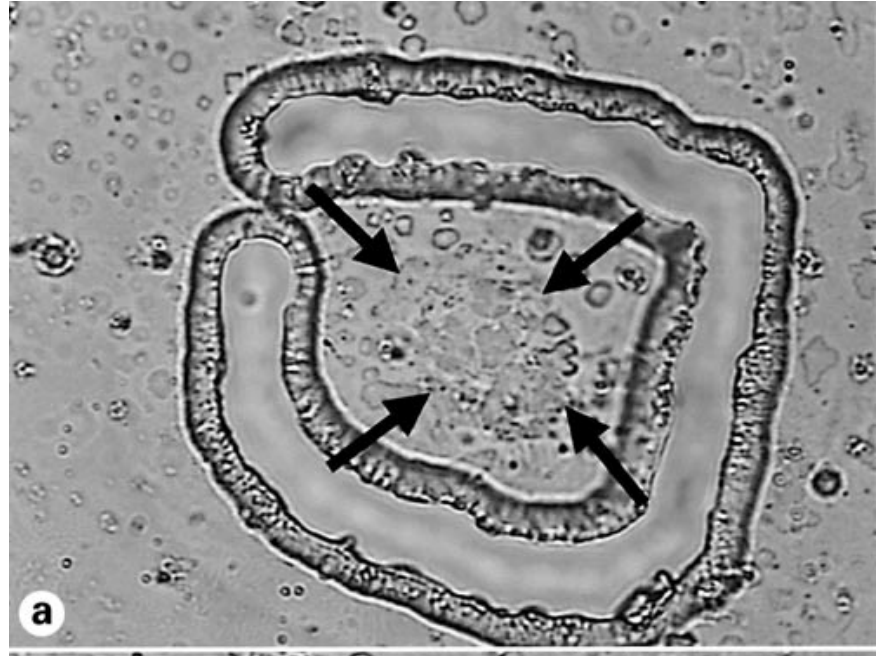

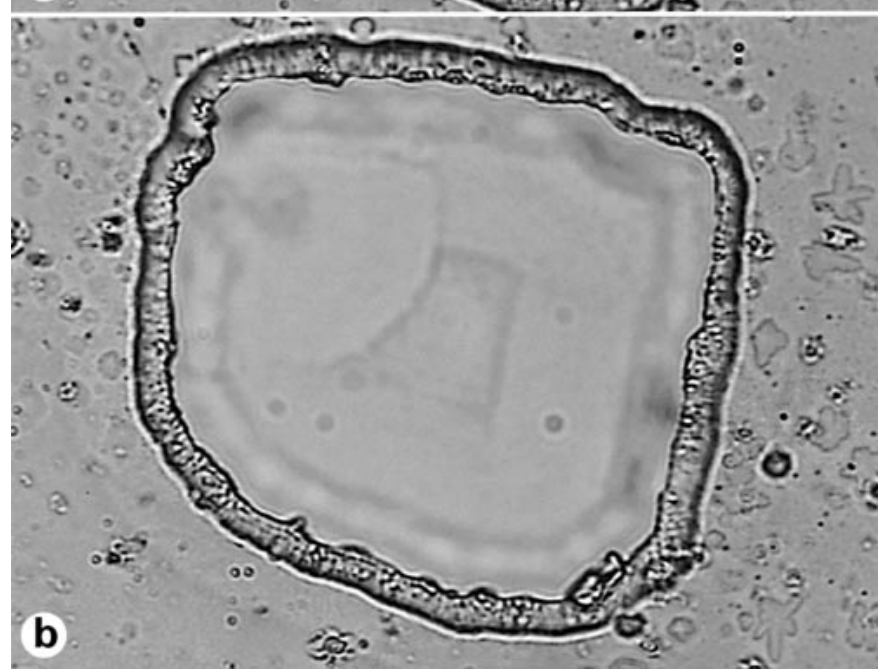

Fig. 4. Laser pressure catapulting of a human PB. a A UV-A laser beam cut the polyethylene membrane around the dried PB (arrows). b After laser pressure catapulting, with one single laser shot, the whole polyethylene membrane fragment was catapulted into the lid of the PCR tube.

Fig. 3. Scheme of PB extraction by a blunt-ended pipette after laser microdissection of the zona pellucida. The extraction pipette can be easily inserted through the laser-drilled hole. 
Table 1. Comparison of $\mathrm{PB}$ extraction with a sharp-ended and a blunt-ended pipette after laser microdissection of the zona pellucida (human)

\begin{tabular}{lll}
\hline Extraction method & $\begin{array}{l}\text { Sharp-ended } \\
\text { pipette only }\end{array}$ & $\begin{array}{l}\text { Laser microdissection } \\
\text { and blunt-ended pipette }\end{array}$ \\
\hline Oocytes total & 93 & 25 \\
Oocytes with extracted first PB & 46 & 18 \\
Oocytes with extracted first and second PB & 47 & 7 \\
Intact oocytes 30 min after manipulation & 67 & 24 \\
Intact oocytes 30 min after manipulation, \% & 72 & 96 \\
\hline
\end{tabular}

For PB removal with the blunt-ended pipette, the zona pellucida of the oocyte was first laser-microdissected with a UV-A laser beam. Therefore, the oocyte was set onto a glass slide in a small drop of manipulation medium. Close to the $\mathrm{PB}$, a hole slightly bigger than the PB was drilled into the zona pellucida (fig. 2) by a high frequency of laser pulses. Therefore, the oocyte was moved forwards and backwards into the laser beam. As the focus of the beam was smaller than $1 \mu \mathrm{m}$, small paths in the zona were visible and allowed precise control of the manipulation procedure. When the hole was big enough, the blunt-ended pipette was introduced through the laser-microdissected hole (fig. 3) and pushed forwards to the PB. Then, the PB was carefully sucked into the pipette and subsequently released into a separate drop of manipulation medium. In a few oocytes we tried to catch single PBs using optical tweezers after zona laser microdissection. The trap was focussed on the PB to drag it out through the previously laser-drilled hole.

For laser pressure catapulting, an extracted PB was washed in a minimum amount of highly purified water and subsequently placed onto a thin polyethylene membrane, which had been mounted onto a glass slide $(0.17 \mathrm{~mm})$. During the drying process, the PB was constantly observed through the microscope to make sure that it could be identified again after drying. After complete drying, the polyethylene membrane around the PB was microdissected by a high frequency series of UV-A laser pulses (fig. 4), leaving a small bridge to avoid uncontrolled loss. With one single, high-energy pulse aimed to the joint, the membrane-bound $\mathrm{PB}$ was catapulted into the lid of a $0.5-\mathrm{ml}$ plastic tube. For this purpose, the inner side of the lid had been covered with $1 \mu \mathrm{l}$ of mineral oil. After catapulting, the membrane fragment stuck to the surface of the paraffin oil and its presence could be verified under a microscope.

\section{Results}

\section{Microdissection of the Zona pellucida}

Laser microdissection of the zona pellucida could easily be performed in all oocytes. In 25 human oocytes, no degeneration was observed after laser microdissection. This was confirmed in other experiments with bovine oocytes, in which 71 oocytes were laser-microdissected with no signs of damage or degeneration.

\section{PB Extraction}

Using a sharp-ended extraction pipette, the first $\mathrm{PB}$ was extracted in 46 oocytes and the first and second $\mathrm{PB}$ in 47 oocytes (table 1 ). Thirty minutes after manipulation, 67 of the 93 manipulated oocytes $(72 \%)$ had remained morphologically intact. After laser microdissection of the zona pellucida in 25 human oocytes, the first PB was extracted in 18 oocytes and the first and second PB in 7 oocytes by a blunt-ended pipette. Twenty-four of the manipulated oocytes ( $96 \%$ ) showed no signs of damage or degeneration $30 \mathrm{~min}$ after PB extraction. In other oocytes, it was possible to use optical tweezers to catch PBs and subsequently move them through the laser-drilled hole out of the perivitelline space.

\section{Laser Pressure Catapulting}

Laser pressure catapulting was applied in six human and fifteen bovine PBs. In all of them, laser microdissection of the polyethylene membrane around the PB was easy to perform and to control. If the laser focus was adjusted to less than $1 \mu \mathrm{m}$, the polyethylene membrane could exactly be microdissected around the PB without touching it. The energy for microdissection was between 1 and $3 \mu \mathrm{J}$. Each PB could subsequently be catapulted into the lid of a PCR tube by a single UV-A shot using 10-fold the energy. Their presence on the surface of the paraffin oil was confirmed in all of the catapulted PB by control under the microscope.

\section{Discussion}

We have been able to show that the combined method of laser microdissection with a UV-A laser and PB extraction with a blunt-ended pipette decreases the degeneration rate of manipulated oocytes in comparison to $\mathrm{PB}$ extraction with a sharp-ended pipette only. Using this method, only $4 \%$ of the oocytes degenerated after manip- 
ulation, whereas $28 \%$ degenerated if a sharp-ended pipette was used for the $\mathrm{PB}$ extraction. In the literature, only results of an overall efficiency after genetic analysis of $\mathrm{PB}$ are given (FISH or PCR-related analysis), ranging between 60 and $82 \%$ [13-15]. Thus, it is impossible to compare our manipulation results with the results of other groups. In our experiments, FISH results of fixed PB can be interpreted in $67 \%$. The overall efficiency, including oocyte degeneration rates, is therefore comparable with the published results of other groups.

The laser trap (laser tweezers) could be used to catch and move $\mathrm{PB}$, and to extract them without pipettes from the oocyte. Although these were the very first trials with a new manipulation method for PB analysis, the first results are very promising. The main advantage of this method is the opportunity to work without any contact. This might dramatically decrease the risk of contamination, which is one of the most demanding problems in subsequent single-cell PCR and further analysis. Extracted PB, bound to a polyethylene membrane, could easily be microdissected and catapulted into PCR tubes. Thus, entire contact-free extraction and further procurement of $\mathrm{PB}$ seem to be probable in the future.

\section{References}

1 Verlinsky Y, Ginsberg N, Lifchez A, Valle J, Moise J, Strom C: Analysis of the first polar body: Preconception genetic diagnosis. Hum Reprod 1990;5:826-829.

2 Verlinsky Y, Cieslak J: Embryological and technical aspects of preimplantation genetic diagnosis; in Verlinsky Y, Kuliev AM (eds): Preimplantation Diagnosis of Genetic Diseases. New York, Wiley-Liss, 1993, pp 49-67.

3 Munné S, Scott R, Sable D, Cohen J: First pregnancies after preconception diagnosis of translocations of maternal origin. Fertil Steril 1998; 69:675-681.

4 Schütze K, Clement-Sengewald A: Catch and move - Cut and fuse. Nature 1994;368:667669.

5 Tadir Y, Wright WH, Vafa O, Ord T, Asch R, Berns MW: Micromanipulation of sperm by a laser generated optical trap. Fertil Steril 1989; 52:870-873.

6 Clement-Sengewald A, Schütze K, Ashkin A, Palma GA, Kerlen G, Brem G: Fertilization of bovine oocytes induced solely with combined laser microbeam and optical tweezers. Hum Reprod 1996;13:259-265.
7 Germond M, Nocera D, Senn A: Improved fertilisation and implantation rates after nontouch zona pellucida microdrilling of mouse oocytes with a $1.48 \mu \mathrm{m}$ diode laser beam. Hum Reprod 1996;11:1043-1048.

8 Liow SL, Bongso A, Ng SC: Fertilization, embryonic development and implantation of mouse oocytes with one or two laser-drilled holes in the zona, and inseminated at different sperm concentrations. Hum Reprod 1996;11: 1273-1280.

9 Antinori S, Selman HA, Caffa B, Panci C, Dani GL, Versaci CL: Zona opening of human embryos using a non-contact UV laser for assisted hatching in patients with poor prognosis of pregnancy. Hum Reprod 1996;1 1:2488-2492.

10 Germond M, Primi MP, Senn A, Pannatier A, Rink K, Delacrétaz G, Montag M, van der Ven H, Mandelbaum J, Veiga A, Barri P: Diode laser for assisted hatching: preliminary results of a multicentric prospective randomized study (abstract). Hum Reprod 1998;13:84-85.
11 Montag M, van der Ven K, Delacrétaz G, Rink $\mathrm{K}$, van der Ven H: Laser-assisted microdissection of the zona pellucida facilitates polar body biopsy. Fertil Steril 1998;69:539-542.

12 Schütze K, Lahr G: Identification of expressed genes by laser-mediated manipulation of single cells. Nat Biotechnol 1999;16:737-742.

13 Rechitsky S, Strom C, Verlinsky O, Amet T, Ivakhnenko V, Kukharenko V, Kuliev A, Verlinsky Y: Accuracy of preimplantation diagnosis of single-gene disorders by polar body analysis of oocytes. J Assist Reprod Genet 1999;16: 169-175.

14 Verlinsky Y, Cieslak J, Ivakhnenko V, Lifchez A, Strom C, Kuliev A: Birth of healthy children after preimplantation diagnosis of common aneuploidies by polar body fluorescent in situ hybridization analysis. Fertil Steril 1996;66: 126-129.

15 Cieslak J, Ivakhnenko V, Evsikov S: Chromosomal abnormalities in first and second polar bodies (abstract). Meeting of the International Working Group on Preimplantation Diagnosis, Bologna, 2000, L-6. 